\title{
Process Control of Pink Guava Puree Pasteurization Process: Simulation and Validation by Experiment
}

\author{
W.M.F. Wan Mokhtar ${ }^{1,2}$, F.S. Taip ${ }^{2}$, N. Abdul Aziz², S.B. Mohd Noor ${ }^{2}$ \\ ${ }^{1}$ Faculty of Food Technology, Universiti Sultan Zainal Abidin, City Campus, Jalan Sultan Mahmud \\ 20400 Kuala Terengganu, Malaysia \\ E-mail:wfadli@unisza.edu.my \\ ${ }^{2}$ Faculty of Engineering, Universiti Putra Malaysia, 43400, Serdang, Selangor, Malaysia \\ E-mail: saleena@eng.upm.edu.my,shekeen@eng.upm.edu.my,samsul@eng.upm.edu.my
}

\begin{abstract}
Recently, process control has been applied extensively in many food processes include pasteurization process. The purpose is to control and maintain the product temperature at desired value. In order to be able to control the process properly, the model of the process needs to be obtained. This research aims to obtain the empirical model and to determine the best control strategy in pasteurization process of pink guava puree. The PID controller tuned by different tuning methods was simulated using Simulink and closed loop responses were observed. Simulation results revealed that PID controller tuned by minimizing of integral absolute error (IAE) method were satisfactory adaptable in this process in term of faster settling time, less overshoot, smallest values of IAE and ISE that less than 1. Then, experiment was performed using this method in order to validate simulation results. In general, a good agreement was achieved between experimental data and dynamic simulation result in control of pasteurization temperature process with $R^{2}=0.83$. As the conclusion, the results obtained can be used as the recommendation for a suitable control strategy for the pasteurization process of pink guava puree in the industry.
\end{abstract}

Keywords - Pasteurization; PID controller; simulation

\section{INTRODUCTION}

Pasteurization is one of food preservation techniques and is widely used in food industries. It involves applying heat to some products such as milk, cream, puree and others at a certain temperature for a certain period of time in order to eliminate harmful pathogenic and spoilage microorganism. As a result, the products can be extending their shelf life and maintain their quality. This process is divided into three section which are heating, cooling and regeneration sections. The most important section is heating which involved heat exchanger equipment to heat up temperature of product at desired set point [1].

Controlling and maintaining the temperature of the process is an important key in pasteurization. Therefore, it needs to design a good control system to control product temperature for preserve the desired product quality. The design good control strategy requires the development of an accurate dynamic model which relates between controlled and manipulated variables. The dynamic model can be developed by theoretically or empirically. Theoretical model is based on fundamentals law as conservation of mass, energy and momentum. This model can be very complex due to consist of many different equations and time consuming. Meanwhile, empirical model is based on real data, easy to develop and suitable for complicated process. Moreover, this model usually sufficient for most food processes such as fermentation [2], drying ([3], [4]), mixing [5] and also pasteurization process [6]. Normally in process control system, the model is in transfer function form which relate between output and input variables. Once the dynamic model has successfully developed, it can give an idea to design a suitable control strategy.

Ilyukhin et al. [7] reported that proportional-integralderivative (PID) controller is commonly used in food industry followed by fuzzy logic techniques and predictive process control. The advantages of PID controller are simplicity, easy to handle and give reasonable performance. There are many literatures have been published regarding with successful implementation of PID controller especially in heat exchanger and pasteurization unit. Maidi et al. [8] and Ramli and Abdullah [9] have found that this controller is gives better performance than adaptive control and PID fuzzy controller in heat exchanger application. On the other 
hand, PID controllers also excellent in other food process such as spray drying as reported by [4]. Their results showed that this controller is not only good in performance criteria, but in robustness stability as well.

However, most of researchers studied and focused on dairy production and water application. There are still lack of literatures that published regarding with process control in beverage product especially pink guava puree. In Malaysia, pink guava (Psidium guajava) is become the important fruit owing to highly potential for export market. The availability throughout the year, high of nutritious and anti - obesity properties can create opportunity for food industries to commercial the varieties of food products from this fruit ([10], [11]). Therefore, the purposes of this study are to simulate dynamic model and investigate the suitable tuning method in PID controller that it satisfies the performance requirements on pasteurization of pink guava puree. Then, the simulation results were validated with experimental data. The best control strategy is suggested to suit in real practical situation of pasteurization process.

\section{MATERIALS AND METHODS}

The dynamic model focuses on pasteurization unit which consist of double tube heat exchanger as shown in Fig. 1. It was specially fabricated in order to mimic the process in industry by LP Equipment Sdn. Bhd., Selangor, Malaysia. The pink guava (Psidium guajava) puree as raw material was obtained from Golden Hope Food and Beverages Sdn. Bhd., Perak, Malaysia. The flow rate for both product and heating medium were constant. The outlet product temperature and inlet heating medium temperature were considered as output and input variables respectively.

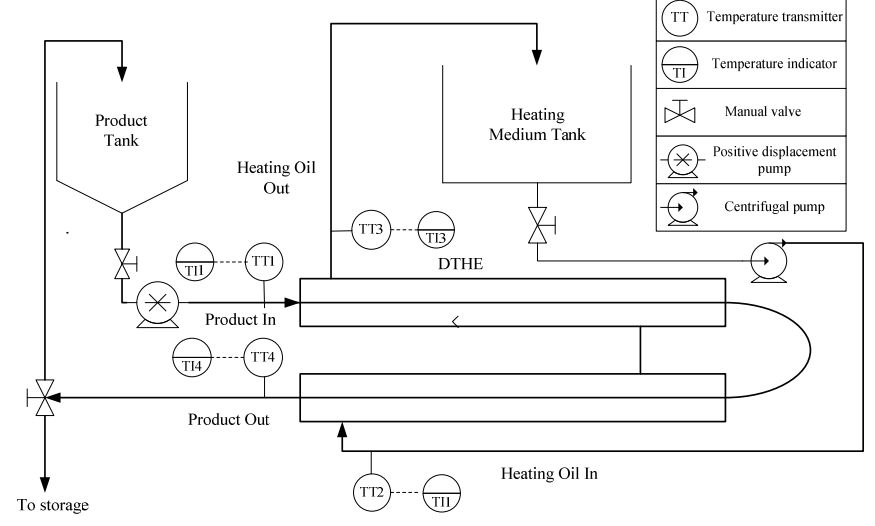

Fig. 1 Process diagram of lab scale pasteurization unit

The data of step change in inlet heating medium temperature from experiment were used in empirical model development. In simulation studies, the feedback control system which PID controller was used for control strategy. The PID were tuned by several tuning methods i.e. ZieglerNichols (T1) [12], simple method (T2) [13], internal model control (T3) [14] and minimization of IAE (T4) [15]. The performances of different tuning methods were determined by using step response into closed loop system. The well known performance criteria such as overshoot, rise time, settling time (based on time required to reach $\pm 95 \%$ of the steady state value), integral of absolute value of error (IAE) and integral of square of error (ISE) were compared for all different tuning methods.

In order to validate simulation results, the control scheme was implementing in lab scale of pasteurization process unit. The data of experiment were recorded for every 0.5 minutes until achieved steady state value.

\section{RESULTS AND DISCUSSION}

\section{A. Dynamic of Empirical Model}

An empirical model of pasteurization process was developed by using data of step change in inlet heating oil temperature. Most of process model can be described with first order plus time delay (FOPTD) model which in transfer function form [16]. The model parameters were calculated using graphical analysis of process reaction curve as reported in ([12], [16]). The process gain, $K_{p}=0.52$ which is ratio of outlet product temperature change to inlet heating medium temperature change. Time delay, $\theta=0.52$ minutes was determined as intersection of tangent with the maximum slope of time axis. Whereas, time constant, $\tau=1.27$ minutes was calculated as time at $63.2 \%$ of output response minus time delay. Then, the model parameters values are substituted into FOPTD model as shown in Equation 1. This obtained model is applicable to apply PID controller since the ratio $(\theta / \tau)$ of obtained model is less than 1 [17].

$$
G(s)=\frac{0.52 e^{-0.52 s}}{1.27 s+1}
$$

\section{B. Simulation Results}

The simulation studies were carried out by using Simulink from MATLAB 2009a software. Four tuning methods were used in simulation to tune PID controller. Table 1 lists tuning parameters values obtained from these tuning methods. As observed in Table 1, T1 and T2 had anticipated generate oscillatory and faster responses due to larger $K_{c}$ and smaller $\tau_{I}$. On the other hand, the controller tuned by T3 and T4 have smaller $K_{c}$ and larger $\tau_{I}$ expected in sluggish and slower responses.

TABLE I

TUNING PARAMETERS FOR PID CONTROLLER

\begin{tabular}{|c|c|c|c|}
\hline $\begin{array}{c}\text { Tuning } \\
\text { method }\end{array}$ & $\begin{array}{c}\text { Controller gain } \\
\left(\boldsymbol{K}_{\boldsymbol{c}}\right)\end{array}$ & $\begin{array}{c}\text { Integral time } \\
\left(\boldsymbol{\tau}_{\boldsymbol{I}}\right)\end{array}$ & $\begin{array}{c}\text { Derivative gain } \\
\left(\boldsymbol{\tau}_{\boldsymbol{D}}\right)\end{array}$ \\
\hline $\mathrm{T} 1$ & 7.05 & 1.30 & 0.21 \\
\hline $\mathrm{T} 2$ & 5.62 & 0.90 & 0.23 \\
\hline $\mathrm{T} 3$ & 1.92 & 1.40 & 0.15 \\
\hline $\mathrm{T} 4$ & 4.37 & 1.79 & 0.20 \\
\hline
\end{tabular}

Fig. 2 shows the open and closed loop responses of PID controller with introduction of step point change from 0 to 1 at time $=0$ minutes. As expected, open loop (OL) which is response without controller did not achieve at desired set point value due to lower of process gain. Meanwhile, closed loop (with controller) had reached the desired steady state value. The factor of larger $K_{c}$ caused $\mathrm{T} 1$ and $\mathrm{T} 2$ resulted oscillatory and aggressive responses as proved in Fig. 2. The same result also reported by [4] in spray dryer application. 
Meanwhile, T3 and T4 resulted in over damped responses before achieve steady state value.

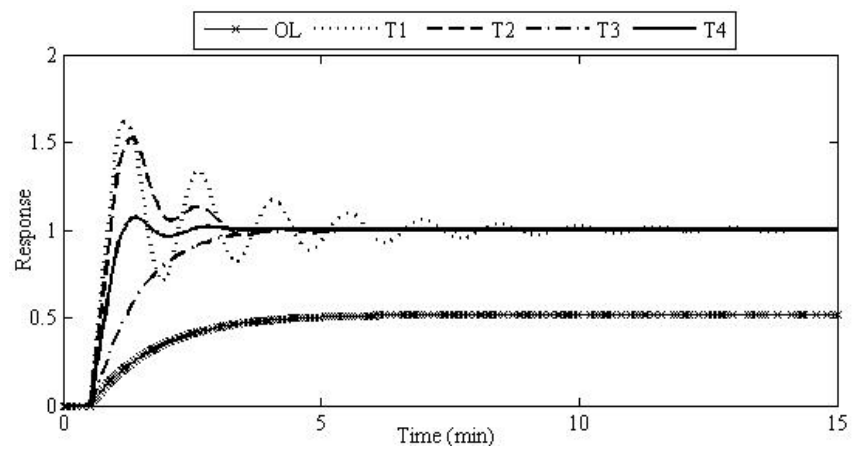

Fig. 2 The process response tuned by different tuning methods

The well known performance criteria viz. overshoot percentage, rise time, settling time, IAE and ISE values are compared in performance analysis and shown in Table 2. From this result, it shows that PID controller tuned by $\mathrm{T} 1$ and T2 give the worst performance due to higher overshoot, OS and larger settling time, $t_{s}$. Hence, these methods are not suitable to implement in real pasteurization process because generate higher overshoot which cause over pasteurized of product. Consequently, the nutritional values of product will destroy as discussed by [18]. T3 method resulted very stable response without overshoot. However, this method has largest value of rise time, $t_{\mathrm{r}}$ which is very sluggish to reach steady state value. From simulation results, the best tuning method is T4 with smaller overshoot, lowest IAE and ISE values and fast in both rise and settling time. This method is most suitable to tune with PID controller in order to implement in control strategy of pasteurization process of pink guava puree.

TABLE 2

PERFORMANCE CRITERIA FOR PID CONTROLLER TUNED BY DIFFERENT TUNING METHODS

\begin{tabular}{|c|c|c|c|c|c|}
\hline $\begin{array}{c}\text { Tuning } \\
\text { method }\end{array}$ & OS (\%) & $\mathbf{t}_{\mathbf{r}}(\mathbf{m i n})$ & $\mathbf{t}_{\mathbf{s}}(\mathbf{m i n})$ & IAE & ISE \\
\hline $\mathrm{T} 1$ & 61.6 & 0.8677 & 7.12 & 1.685 & 0.892 \\
\hline $\mathrm{T} 2$ & 52 & 0.9299 & 3.04 & 1.196 & 0.804 \\
\hline $\mathrm{T} 3$ & 0 & 4.5610 & 3.00 & 1.426 & 1.009 \\
\hline $\mathrm{T} 4$ & 7.1 & 1.1720 & 1.55 & 0.874 & 0.714 \\
\hline
\end{tabular}

\section{Simulation Validation}

In this section, experimental work was carried out to validate the simulation results. The best tuning method determined by simulation studies which is T4 was applied in process control of lab scale pasteurization unit. The tuning parameters of T4 were set in control panel of system. Fig. 3 shows the closed loop response of experiment and simulation for set point change from $45^{\circ} \mathrm{C}$ to $48^{\circ} \mathrm{C}$ at time $=12$ minutes. As we can see, the dynamic response of simulation was faster than experiment result due to approximation of time delay in simulation work. In simulation, Padé approximation is used to estimate the time delay response and it become quite shorter than experimental value. Moreover, time required by heater to heat up the product cause the dynamic response of experiment become slower. However, simulation result remains demonstrates good agreement with the experiment result with $R^{2}=0.83$. It is suggests that PID controller tuned by T4 method is most suitable used in pasteurization process control especially for pink guava puree product.

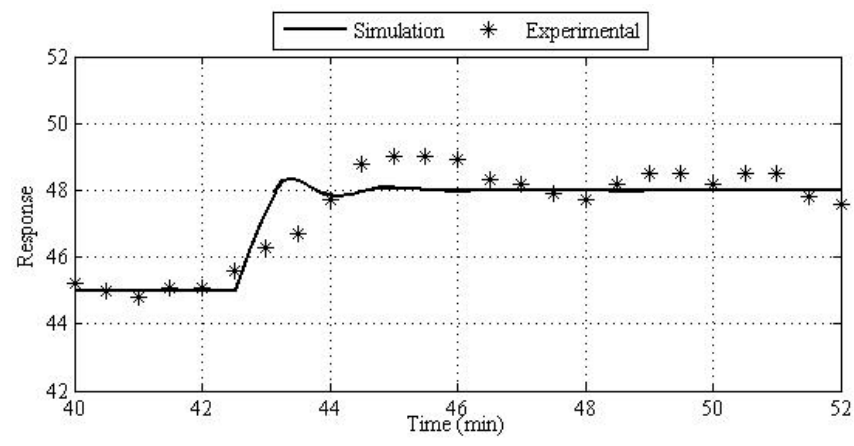

Fig. 3 Simulation and experiment results of pasteurization process

\section{CONCLUSION}

The empirical model of pasteurization process was obtained in first order plus time delay model. Simulation results revealed that $\mathrm{T} 4$ method using by PID controller is most suitable in pasteurization process of pink guava puree due to best achievable controller performances. Then, simulation results were validated by experimental work. In general, a good agreement was achieved between experimental data and simulation result with $R^{2}=0.83$.

\section{ACKNOWLEDGMENT}

The authors would like to acknowledge Ministry of Higher Education (MOHE) Malaysia, Universiti Sultan Zainal Abidin and Universiti Putra Malaysia for funding under Fundamental Research Grant Scheme (Grant No. 5523719).

\section{REFERENCES}

[1] G. D. Saravacos, and A. E. Kostaropoulos, Handbook of Food Processing Equipement. New York: Kluwer Academic / Plenum Publishers, 2002.

[2] P. Potočnik, and I. Grabec, "Empirical modelling of antibiotic fermentation process using neural networks and genetic algorithms," Mathematics and Computers in Simulation, vol. 49, pp. 363 - 379, 1999.

[3] A. Khoshhal, A. A. Dakhel, A. Etemadi, and S. Zereshki, "Artificial neural network modeling of apple drying process," Journal of Food Process Engineering, vol. 33, pp. 289 - 313, 2010.

[4] L. W. Tan, F. S. Taip, and N. A. Aziz, "Simulation and control of spray drying using nozzle atomizer spray dryer," International Journal of Engineering and Technology, vol. 9 (10), pp. 12 - 17, 2009.

[5] J. A. V. Selvi, T. K. Radkakrishnan, and S. Sundaram, "Performance assessment of PID and IMC tuning methods for a mixing process with time delay," ISA Transactions, vol. 46, pp. 391 - 397, 2007.

[6] J. J. Ibarrola, J. C. Guillén, J. W. Sandoval, and M. Garćia-Sanz, "Modelling of a high temperature short time pasteurization process," Journal of Food Control, vol. 9 (5), pp. 267 - 277, 1998.

[7] S. V. Ilyukhin, T. A. Haley, and K. S. Rajesh, "A survey of automation practices in food industry," Food Control, vol. 12, pp. $285-296,2001$.

[8] A. Maidi, M. Diaf, and J. P. Corriou, "Optimal linear PI fuzzy controller design of a heat exchanger," Chemical Engineering and Processing, vol. 47, pp. 938 - 945, 2008. 
[9] N. M. Ramli, and B. Abdullah, "Modeling and advanced regulatory control of a heat exchanger. Proc. SOMCHE, 2007.

[10] M. Y. Ayub, M. N. Norazmir, S. Mamot, K. Jeeven, and H. Hadijah, H. "Anti-hypertensive effect of pink guava (Psidium guajava) puree on spontatenous hypertensive rats," International Food Research Journal, vol. 17, pp. 89 - 96, 2010.

[11] M. N. Norazmir, and M. Y. Ayub, "Beneficial lipid-lowering effects of pink guava puree in high fat diet induced-obese rats," Malaysia Journal of Nutritional, vol. 16 (1), pp. 171 - 185, 2010.

[12] C. A. Smith, and A. B. Corropio, Principle and practice of automatic control, 3rd ed., New York: John Wiley, 2006.

[13] R. P. Sree, M. N. Srivinas, and M. Chidambaram, "A simple method of tuning PID controllers for stable and unstable FOPTD systems," Computers and Chemical Engineering, vol. 28, pp. 2201 - 2218, 2004.
[14] R. C. Panda, "Synthesis of PID tuning rule using the desired closedloop response," Indian Engineering Chemical Resources, vol. 47, pp. $8684-8692,2008$

[15] C. R. Madhuranthakam, A. Elkamel, and H. Budman, "Optimal tuning of PID controllers for FOPTD, SOPTD and SOPTD with lead processes," Chemical Engineering and Processing: Process Intensification, vol. 47 (2), pp. 251 - 264, 2008.

[16] T. E. Marlin, Process control: designing processes and control systems for dynamic performance, United State of America: McGraw - Hill, 2000.

[17] W. Tan, J. Liu, T. Chen, and H. J. Marquez, "Comparison of some well-known PID tuning formulas," Computers and Chemical Engineering, vol. 30, pp. 1416 - 1423, 2006.

[18] A. P. M. Hasting, "Practical considerations in the design, operation and control of food pasteurization processes," Food Control, pp. 27 32, 1992. 